\title{
COMPUTATIONAL MODELING OF THE MECHANICS OF ENERGY HARVESTERS BASED ON TENSEGRITY SOLAR FAÇADES
}

\author{
RAFFAELE MIRANDA ${ }^{1}$, ENRICO BABILIO ${ }^{2}$, NARINDER SINGH ${ }^{3}$, \\ FILIPE SANTOS ${ }^{4}$, FERNANDO FRATERNALI ${ }^{5}$ \\ ${ }^{1}$ Department of Civil Engineering, University of Salerno \\ Via Giovanni Paolo II 132, 84084 - Fisciano (SA), Italy \\ ramiranda@unisa.it
}

${ }^{2}$ Department of Structures for Engineering and Architecture (DiSt), University of Naples "Federico II" via Forno Vecchio 36, 80134 - Naples, Italy

enrico.babilio@unina.it

${ }^{3}$ Department of Civil Engineering, University of Salerno

Via Giovanni Paolo II 132, 84084 - Fisciano (SA), Italy snarinder@unisa.it

${ }^{4}$ FCT, Civil Engineering Department, Universidade NOVA de Lisboa

Campus da Caparica 2829-516 Caparica, Portugal fpas@fct.unl.pt

${ }^{5}$ Department of Civil Engineering, University of Salerno

Via Giovanni Paolo II 132, 84084 - Fisciano (SA), Italy

f.fraternali@unisa.it

Key words: Tensegrity structures, dynamic solar façades, mechanical energy harvesters

\begin{abstract}
This work is focused on the computational design of tensegrity shading systems of energy efficient buildings which mitigate air conditioning consumption and optimize the energy performance of the building. It is concerned with the design of active solar façade screens based on lightweight tensegrity units, which are easily integrated with energy harvesting piezoelectric cables. The unit cells of the analyzed screens are controlled by tensioning and releasing selected cables of the structure. A numerical procedure simulates the dynamics of the analyzed tensegrity façades, by considering the opening and closure motions of the screens, and the vibrations produced by the action of dynamic wind forces. The energy harvesting ability of the proposed façade is numerically estimated.
\end{abstract}

\section{INTRODUCTION}

The design of sustainable buildings naturally leads to analyze the fundamental elements of such buildings, which consist of natural or mechanic ventilation systems, protective shading systems, and, eventually, home automation systems and renewable energy harvesting devices.

Among the objectives of the EU's Horizon 2020 program on the "Secure, Clean and 
Efficient Energy" (SC3) social challenge is to reduce energy consumption and carbon emissions through intelligent and sustainable use in order to pursue a more ambitious energy policy and maintain current living standards while reducing energy dependence on fossil fuels [1]. The demand for energy savings and the excessive $\mathrm{CO}_{2}$ emissions generated by buildings has called for the use of new "interactive building envelopes", defined as systems that interacts with the variations of the external climatic conditions by means of automatic control devices, optimizing the environmental performance of the internal microclimate.

This paper presents a mechanical study of dynamic sunscreens inspired to Al Bahar Towers facade in Abu Dhabi [2,3]. We enrich the kinematical study presented in [4] by providing a procedure for the implementation of the system and the study of its dynamics under wind forces. We employ such a procedure for the simulation of the motion exhibited by origami sunscreens under fluctuating (positive/negative) wind forces. The wind-induced energy stored in the activation strings is investigated with the aim of exploring the potential of the analyzed sunscreens as wind power harvesters. Their energy harvesting capacity is compared with that of microeolic turbines available in the market [5-7]. The results show that tensegrity solar façades as wind power harvesters are highly feasible and may lead to design of cost-effective shading systems.

\section{WTABS MODEL}

In the present study a tensegrity module, addressed as 'wind tensegrity Al Bahar screen' improvement of TABS Al Bahar towers in Ab bars and 3 strings. The string elements [4]. In order to harvest mechanie with a number of D-bal
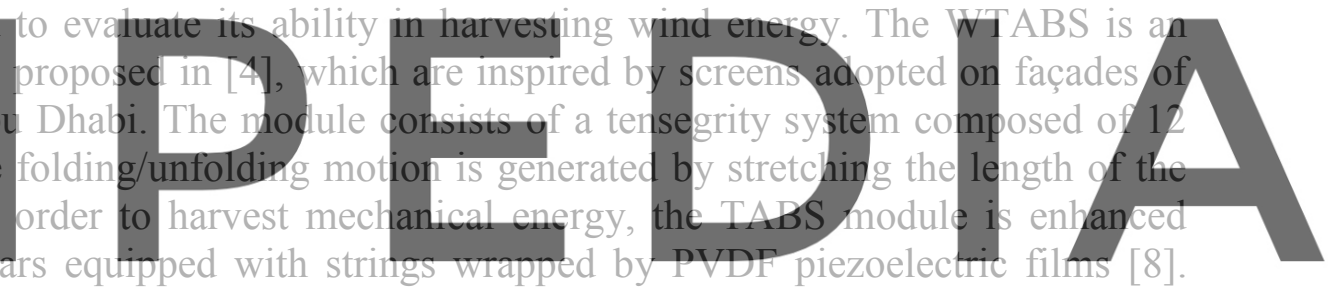

Figure 1 reports the main elements composing WTABS. In particular, Fig. 1(a) describes the

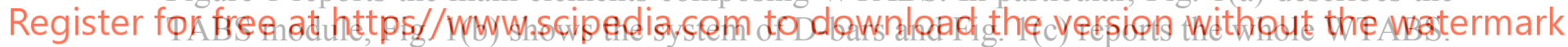

The actuation mechanism is generated by a piston Rolaram ${ }^{\circledR}$ R2501190, with design characteristics of dynamic loading equal to $294 \mathrm{kN}$ and a maximum stroke of $3500 \mathrm{~mm}$ [9], which is located on the highest point of the WTABS structure. Such a piston stretches and releases the perimetral strings, thus activating the dynamic motion. A telescopic sliding constraint positioned behind the structure adjusts the scrolling along the $z$ axis, while two linear springs, with stiffness constant $K=67 \mathrm{kN} \mathrm{m}^{-1}$, control the in-plane movements of the other vertices.
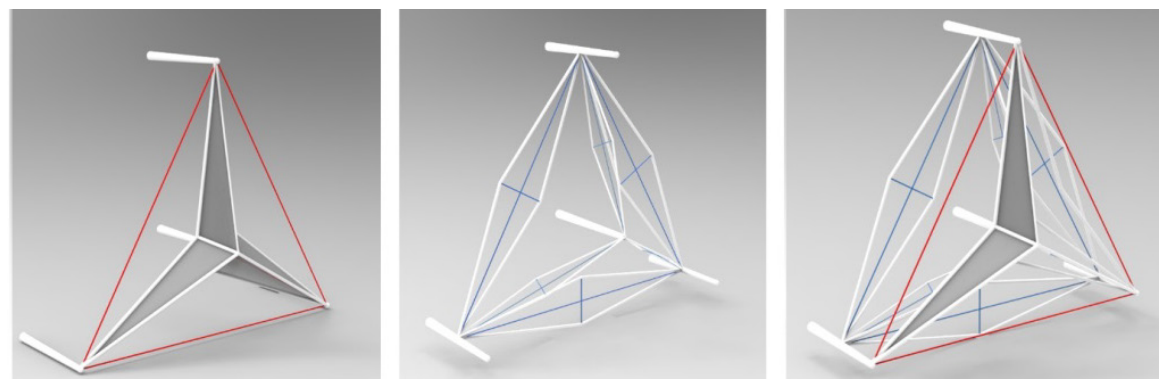

Figure 1: WTABS system: TABS model configuration, left; D-bar elements, middle; WTABS assembly, right. 


\section{COMPUTATIONAL MODELING OF THE WTABS DYNAMICS}

This Section is devoted to introduce the dynamic equations of the tensegrity sunscreen module. We will indicate matrices with bold capital letters (i.e. $\mathbf{X}$, but in case of diagonal matrices indicated by hatted italic letters, i.e. $\hat{x}$ ), vectors with bold lower case letters (i.e. $\mathbf{x}$ ), scalars with italic letters (i.e. $x$ ).

We consider an algorithm for the rigid body dynamics of a general tensegrity network made of $n_{n}$ nodes, $n_{b}$ bars and $n_{s}$ strings. The algorithm includes a rigidity constraint on the deformation of the bars.

By defining the generic node $i$, with $i \in\left[1, \ldots, n_{n}\right]$, located in the three-dimensional Euclidean space by the vector $\mathbf{n}_{i} \in \mathbb{R}^{3}$, we can introduce the following nodal matrix

$$
\mathbf{N}=\left[\begin{array}{llllll}
\mathbf{n}_{1} & \mathbf{n}_{2} & \ldots & \mathbf{n}_{i} & \ldots & \mathbf{n}_{n_{n}}
\end{array}\right] \in \mathbb{R}^{3 \times n_{n}} .
$$

The external force vector $\mathrm{w}_{i} \in \mathbb{R}^{3}$, acting on the $i^{\text {th }}$ node and including the wind forces, gives us the external force matrix

$$
\mathbf{W}=\left[\begin{array}{llllll}
\mathbf{W}_{1} & \mathbf{W}_{2} & \ldots & \mathbf{W}_{i} & \ldots & \mathbf{W}_{n_{n}}
\end{array}\right] \in \mathbb{R}^{3 \times n_{n}} .
$$

\section{The matrices $\mathbf{B}, \mathbf{S}$ and $\mathbf{R}$ of bars, strings and center of mass are defined as}
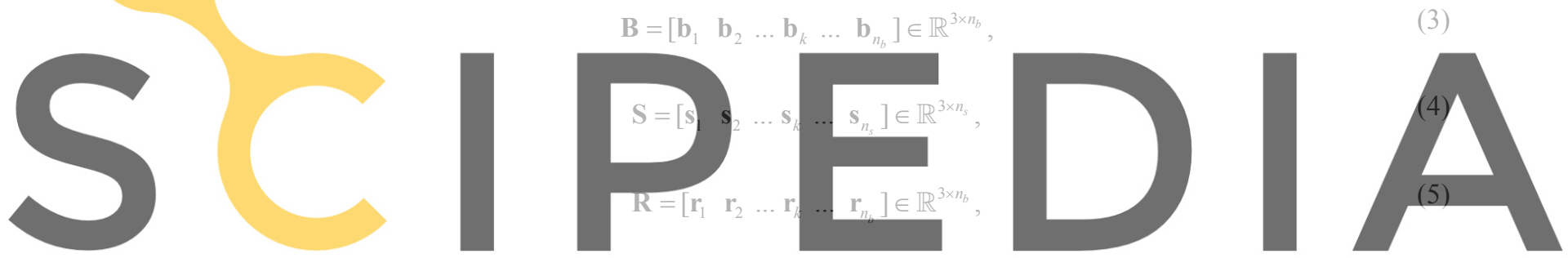

where $\mathbf{b}_{\mathrm{k}}, \mathbf{s}_{\mathrm{k}}$, and $\mathbf{r}_{k}=\left(\mathbf{n}_{i}+\mathbf{n}_{j}\right) / 2$ defines the vectors of respectively $k^{\text {th }}$ bar, $k^{\text {th }}$ string and center

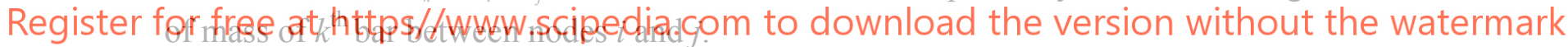

Defining $\mathrm{C}_{\mathrm{B}} \in \mathbb{R}^{n_{3} \times n_{n}}, \mathrm{C}_{\mathrm{S}} \in \mathbb{R}^{n_{3} \times n_{n}}$ and $\mathrm{C}_{\mathrm{R}} \in \mathbb{R}^{n_{3} \times n_{n}}$ as the connectivity matrices of bars, cables and center of mass, respectively, we can rewrite the above matrices as

$$
\begin{aligned}
& \mathbf{B}=\mathbf{N} C_{\mathbf{B}}^{\mathrm{T}}, \\
& \mathbf{S}=\mathbf{N C}_{\mathrm{S}}^{\mathrm{T}}, \\
& \mathbf{R}=\mathbf{N C}_{\mathbf{R}}^{\mathrm{T}} .
\end{aligned}
$$

It is worth stressing that the general element $\mathbf{C}_{\mathbf{B} i j}$ (or $\mathbf{C}_{\mathbf{S} i j}$ ) is equal to -1 if vector $\mathbf{b}_{i}$ (or $\left.\mathbf{s}_{i}\right)$ is directed away from node $j^{\text {th }}$, to 1 if vector $\mathbf{b} i$ (or $\mathbf{s}_{i}$ ) is directed toward node $j^{\text {th }}$, and to 0 if vector $\mathbf{b}_{i}$ (or $\mathbf{s}_{i}$ ) does not touch node $j$. Therefore we can describe the class number of a tensegrity network and state that, in this case, we are considering a class 6 tensegrity structure (we recall that a tensegrity system is of class $m$ if the maximum number of bars concurring in each node is equal to $m$ ).

By introducing the force density parameter of the $k^{\text {th }}$ string as 


$$
\gamma_{k}=\max \left[0, k_{k}\left(1-\frac{L_{k}}{s_{k}}\right)\right]+\gamma_{c k}
$$

being $k_{k}$ the material stiffness coefficient, $L_{k}$ the rest length, $s_{k}$ the actual length and $\gamma_{c k}$ the force density due to damping given by

$$
\begin{aligned}
& \gamma_{c k}=c_{k} \frac{s_{k}}{s_{k}} \text {, if } s_{k} \geq L_{k} \text {, } \\
& \gamma_{c k}=0, \quad \text { if } s_{k}<L_{k} \text {, }
\end{aligned}
$$

where $\dot{s}_{k}$ is the norm of the velocity vector of the string, we can write the equations of motion of a class 1 tensegrity system as follows [10]

$$
\ddot{N} \mathbf{M}+\mathbf{N K}=\mathbf{W}
$$

Here, superimposed dots stand for derivative with respect to time and mass and stiffness matrices read as

$$
\mathbf{M}=\frac{1}{12} \mathbf{C}_{\mathbf{B}}^{\mathrm{T}} \widehat{m} \mathbf{C}_{\mathbf{B}}+\mathbf{C}_{\mathbf{R}}^{\mathrm{T}} \widehat{m} \mathbf{C}_{\mathbf{R}} \in \mathbb{R}^{n_{n} \times n_{n}},
$$

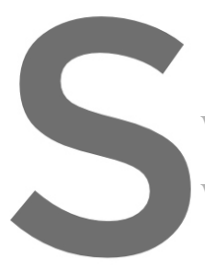

with $\hat{m} \in \mathbb{R}^{n_{b} \times n_{b}}, \hat{\gamma} \in \mathbb{R}^{n_{3} \times x_{n}}$ and $\hat{\lambda} \in \mathbb{R}^{n^{\prime}}$
while $\hat{\gamma}$ and $\hat{\lambda}$ collect string and
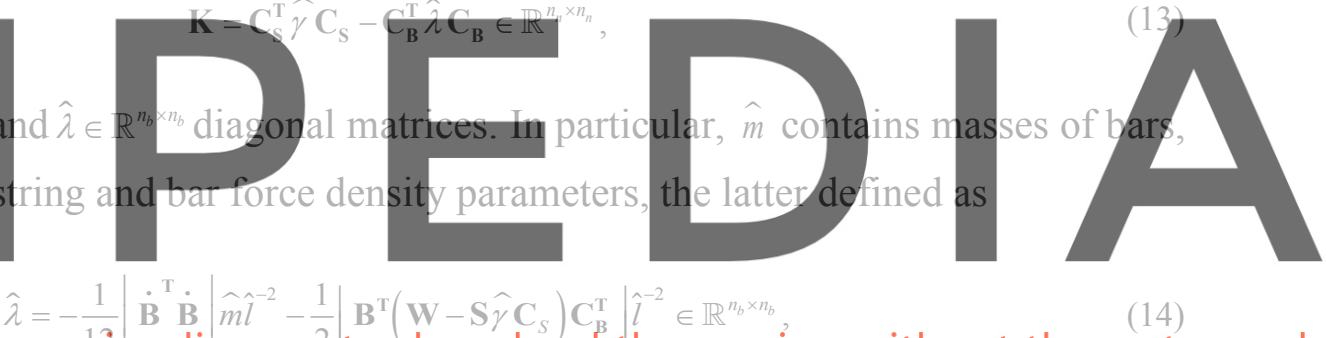

(14)

Register for free at https//www.scipedia.com to download the version without the watermark

where $\hat{l}^{-2} \in \mathbb{R}^{n_{b} \times n_{b}}$ is a diagonal matrix of terms $l_{k}^{-2}=\left\|\mathbf{b}_{k}\right\|^{-2}$ and $\lfloor\cdot\rfloor$ stands for a diagonal matrix of its arguments.

In the field of form finding tensegrity methods, the force density method is used to convert the nonlinear equilibrium systems into linear ones through the transformation between force density (the control variable that appears linearly in the system) and rest length [11].

To integrate the equations of motion (11) we use the fourth-order Runge-Kutta formula $[12,13]$ considering a fixed integration time step.

\section{NUMERICAL APPLICATIONS}

The present Section is focused on numerical results of the shading system WTABS. The dynamics of the system and the corresponding amount of convertible mechanical energy are investigated under two basic processes, namely motion driven by ram activation, taking place at eye opening or closure, and wind fluctuations, due to wind gusts loading the deployed screen. In simulation, we consider a damping factor of 5\% and a time step of $0.025 \mathrm{~s}$. 


\subsection{Simulation of the activation motion of the WTABS module}

In order to analyze the dynamical response and compute the amount of convertible mechanical energy from strings we assume the screen is activated by a force acting on a node, that we call as the actuated node, impressed by the linear actuator Rolaram ${ }^{\circledR}$ R2501190 [9]. The loading program, divided in two phases of 40 seconds each, consists in deploying the screen from the initial state to the almost flat configuration, by linearly increasing over time the actuating force, and then maintaining the screen deployed, with the force held constant over time.

At $t=0 \mathrm{~s}$, the force on the actuated node has value $F_{\min }=72.690 \mathrm{kN}$, necessary to sustain the initially pre-stressed configuration. At $t=20 \mathrm{~s}$, the external force reaches its maximum value, set to $F_{\max }=276.221 \mathrm{kN}$.

Notice that, in order to save energy in an actual application, when the screen is folded or deployed at the desired configuration, the actuated node could be locked with a releasable hook and the actuator could be switched off. Whenever the screen configuration must be changed, actuator could be switched on and the hook released.

The initial configuration of the system corresponds to the screen almost folded, with initial velocities set to zero and boundary strings under tensile stress, which takes value

$$
N_{0}=\frac{E_{S} A_{S}}{L_{0}}\left(L_{i}-L_{0}\right)=11846.6 \mathrm{~N},
$$

where $E_{S}$ and $A_{S}=\pi(d / 2)^{2}$ are effective Young's modulus and cross-sectional area of the

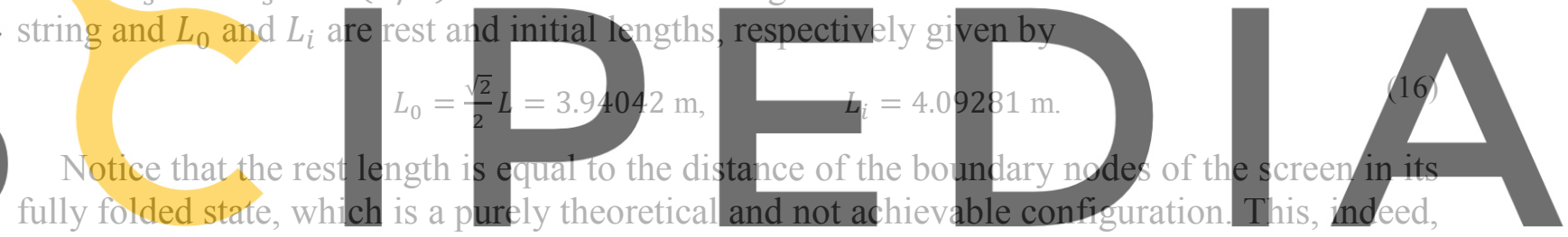

ensures that boundary strings in actual applications cannot be stress-free.

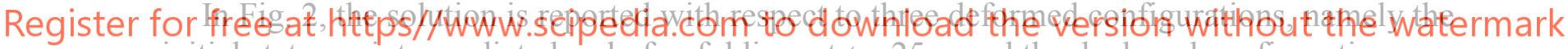
initial state, an intermediate level of unfolding, at $t=25 \mathrm{~s}$, and the deployed configuration.
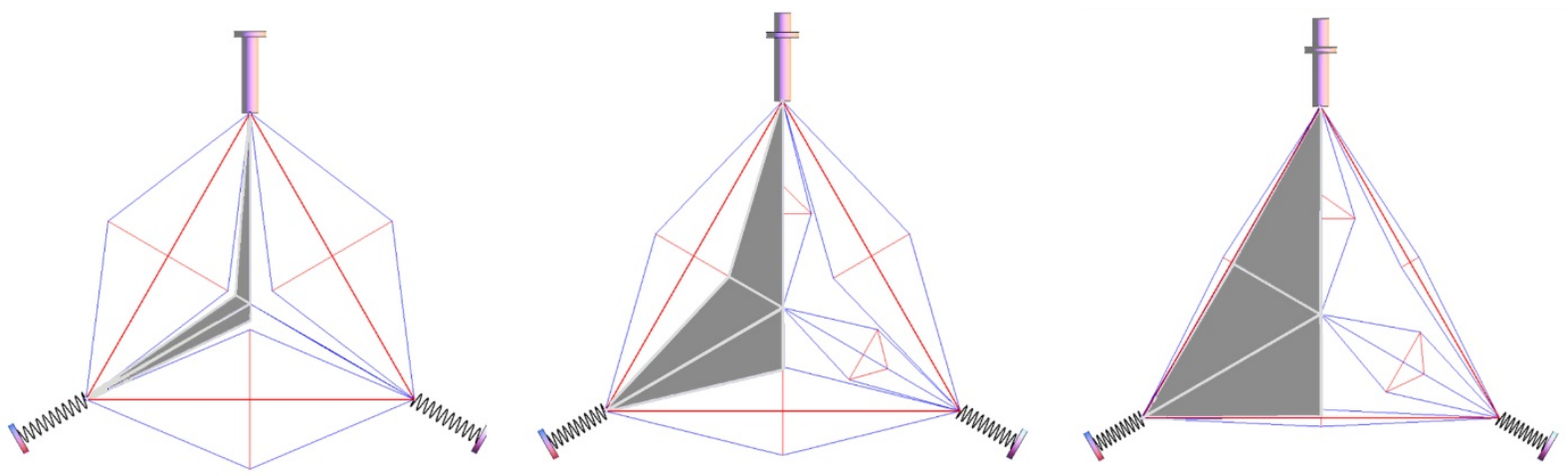

Figure 2: Three configurations of screen (gray shaded) and underlying assemblage of D-bars. From the left: at $t$ $=0 \mathrm{~s}, t=25 \mathrm{~s}$, and $t=50 \mathrm{~s}$. The boundary springs and the actuator ram are represented with amplified scales. 
Finally, strains into strings will be used to compute the mechanical energy transmitted to the covering piezoelectric film in following Sect. 5.

\subsection{Modeling of the wind forces}

The instantaneous wind vector speed $\mathbf{v}$ can be represented by the composition of the average speed $\mathbf{V}_{m}$ over an interval of $10 \mathrm{~min}$, characterized by long-term variations, and the atmospheric turbulence $\mathbf{v}^{\prime}$, with high frequency fluctuations [14]

$$
\begin{gathered}
\mathbf{V}(P, t)=\mathbf{V}_{m}(P)+\mathbf{V}^{\prime}(P, t), \\
\mathbf{V}_{m}(P)=\mathbf{i} \cdot v_{m}(z), \\
\mathbf{V}^{\prime}(P, t)=\mathbf{i} \cdot v_{1}^{\prime}(P, t)+\mathbf{j} \cdot v_{2}^{\prime}(P, t)+\mathbf{k} \cdot v_{3}^{\prime}(P, t),
\end{gathered}
$$

where $P$ is any point at the height $z$ on the ground, $t$ is the time, $\mathbf{i}, \mathbf{j}, \mathbf{k}$ are the unit vectors along axes $x, y, z ; v_{m}$ is the average speed along $x$ and $v_{1}^{\prime}, v_{2}^{\prime}, v_{3}^{\prime}$ are the longitudinal $(x)$, lateral $(y)$ and vertical $(z)$ components of turbulence.

In what follows we consider only turbulent fluctuations $v_{1}^{\prime}$ and assume they are representable as a single stationary Gaussian random process, having zero mean, depending only on the height and time, that is $v_{1}^{\prime}(P, t)=v^{\prime}(z, t)$, and specified by the spectral density [14]
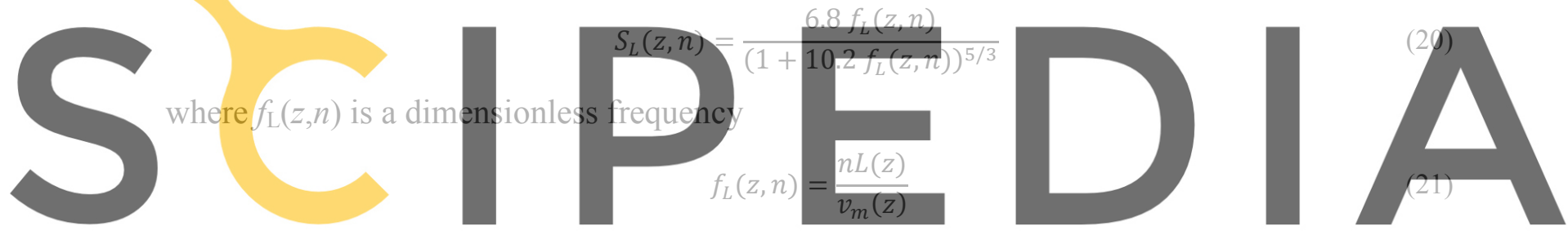

being $L(z)$ the turbolence scale and $n$ the natural frequency.

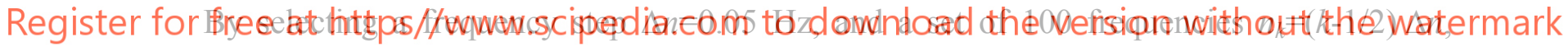
$k=1, \ldots, 100$, we generate a sample of the variable wind speed using the superimposition method with sinusoidal functions, that is [15]

$$
v^{\prime}(z, t)=\sum_{k=1}^{N} \sqrt{2 S_{L}\left(z, n_{k}\right) \Delta n} \cos \left(2 \pi n_{k} t+\varphi_{k}\right)
$$

with $\varphi_{k}$ randomly generated phase angles, in rad, over the range $0-2 \pi$. Finally, the peak value of the wind kinetic pressure, in $\mathrm{m} / \mathrm{s}$, is expressed by

$$
q_{p}(z, t)=\frac{1}{2} \rho\left(v_{m}(z)+v^{\prime}(z, t)\right)^{2}
$$

being $\rho=1.25 \mathrm{~kg} \mathrm{~m}^{-3}$ the density of the air.

For the sake of computation, we assume data are compatible with Al Bahar Towers, that is height $z^{*}=120 \mathrm{~m}$ in a caostal area exposed to wind, leading to the turbolence scale $L\left(z^{*}\right)=40.1589 \mathrm{~m} \mathrm{[14]}$. Further, to simulate the wind pressure of $3.5 \mathrm{kPa}$ obtained in wind 
tunnel tests on full-scale screen prototypes presented in [3] we consider the average velocity $v_{m}\left(z^{*}\right)=73.4 \mathrm{~m} \mathrm{~s}^{-1}$.

The wind force over the generic infill panel is assumed acting along its normal [4]. Initial configuration coincides with the deployed state after the actuation process and initial nodal velocities are set to zero. Total time of computation is $250 \mathrm{~s}$.

Strains into strings induced by wind fluctuations will be used to compute the harvested energy in following Sect. 5.
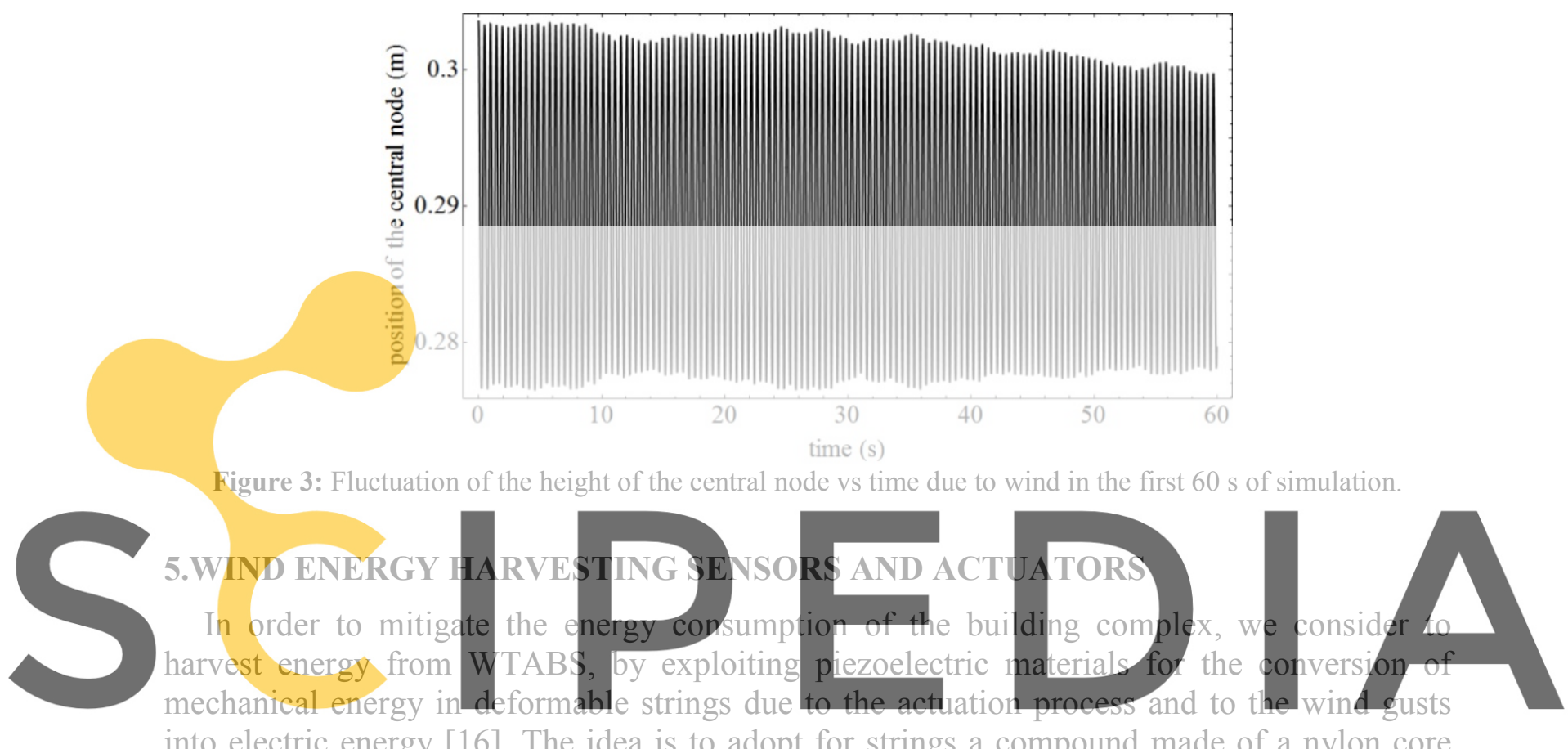
into electric energy [16]. The idea is to adopt for strings a compound made of a nylon core

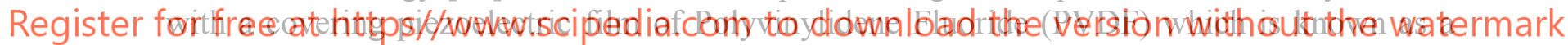
highly sensitive piezoelectric material.

The mechanical energy stored $W_{M}$ into the film is converted in electric energy $W_{E}$ through (see [17], p. 21)

$$
W_{E}=k_{33}^{2} W_{M}
$$

where $k_{33}$ is the piezoelectric coefficient representing the ability of the device mechanically strained in longitudinal direction of the generic strings (which is addressed as the local direction 3), to transform mechanical energy into electrical energy (and vice versa). To compute $W_{M}$ we consider strains into strings obtained from numerical computations and the Young's modulus $E_{f}$ of PVDF, reported in Table 1, along with other relevant properties of the film (parameters taken from [18], p. 79, except $k_{33}$, from [19], p. 125).

Table 1: Properties of PVDF in the direction of stress

\begin{tabular}{llll}
\hline Young's modulus & Ultimate stress & Ultimate strain & Coupling coefficient \\
$\mathrm{E}_{\mathrm{f}}$ & $\sigma_{\mathrm{f}}$ & $\varepsilon_{\mathrm{f}}$ & $k_{33}$ \\
$(\mathrm{GPa})$ & $(\mathrm{MPa})$ & $(\%)$ & $(-)$ \\
\hline 2.7 & 350 & 16.9 & 0.15 \\
\hline
\end{tabular}


In order to evaluate $W_{M}$ to be converted in $W_{E}$ during the actuation process and wind fluctuations we can estimate to total amount of internal energy as

$$
W_{M}=\frac{1}{2 T} \sum_{s=1}^{n_{s}}\left(E_{f} A_{f} L_{i s} \int_{0}^{T} \varepsilon_{s}(t)^{2} \mathrm{~d} t\right)
$$

where $T$ is the total time of simulation, $L_{i s}$ is the initial length of the $s^{\text {th }}$ string, $n_{s}$ is the total number of strings with piezoelectric ability, and $A_{\mathrm{f}}$ is the area of the covering film with a thickness of $0.1 \mathrm{~mm}$.

During the first process we have considered, namely a simulation of the activation process followed by a hold-on phase of a total time $T=80 \mathrm{~s}$, the mechanical energy and its electrical counterpart take values

$$
\begin{aligned}
& W_{M}^{\text {act }}=2532.757 \mathrm{~kJ}=701.044 \mathrm{Wh}, \\
& W_{E}^{\text {act }}=57.784 \mathrm{~kJ}=15.7735 \mathrm{Wh} .
\end{aligned}
$$

In the simulation of wind, we considered a total time $T=250 \mathrm{~s}$. The energies obtained are

$$
\begin{gathered}
W_{M}^{\text {wind }}=4.595 \times 10^{-2} \mathrm{~kJ}=1.276 \mathrm{Wh} \times 10^{-2}, \\
W_{E}^{\text {wind }}=1.034 \times 10^{-4} \mathrm{~kJ}=2.872 \times 10^{-4} \mathrm{Wh} .
\end{gathered}
$$

One observes that the overall electric energy from WTABS mounted on a façade like that of Al Bahhar towers during actuation is approximately equal to that produced panels and 87 microel analyzed sunscreen can power can be usefully 6.
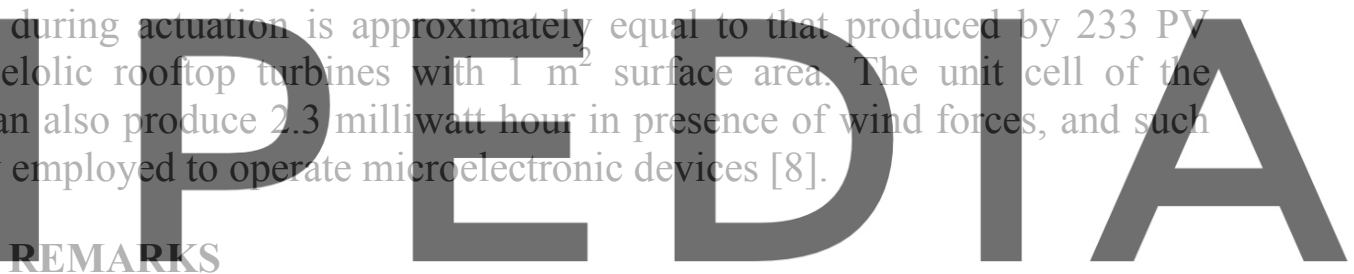

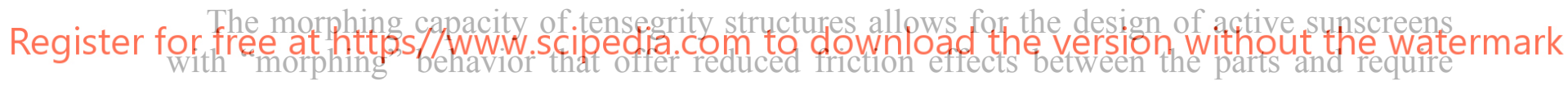

limited energy consumption for their implementation. The modification of their configuration during the hours of the day can be easily achieved by regulating the tension in the cables. The compressed elements of the screens remain undeformed during the opening and closing motions and can therefore be equipped with solar panels and/or rigid membranes. The present work has provided comprehensive computational models in matrix and vector forms for the simulation of the dynamics of dynamic solar façades with a tensegrity-based origami architecture. The special ability of such structures to serve as energy harvesters of new generation energy efficient buildings has been diffusely discussed, by considering their actuation motion and wind induced fluctuations.

Future studies will be focused on the design of modeling of dynamic facades to develop hybrid structures based on natural ventilation and solar shading elements. Energy harvesting research will be directed towards the use of various piezoelectric elements, such as ceramics, composites, monocrystals and polymers, for the electrical conversion of the energy. The experimental validation of the analyzed sunscreens will be conducted on reduced scale physical models through structural identification procedures [20]. 


\section{ACKNOWLEDGEMENTS}

R.M., N.S., and F.F. acknowledge financial support from MIUR under the PRIN 2017 National Grant 'Multiscale Innovative Materials and Structures' (grant number 2017J4EAYB).

\section{REFERENCES}

[1] European Commission, HORIZON 2020 Work Programme 2014-2015, PART 5.ii, p. 98, 2014.

[2] Armstrong, A., Buffoni, G., Eames, D., James, R., Lang, L., Lyle, J. and Xuereb, K. The Al Bahar towers: multidisciplinary design for middle east high-rise. Abu Dhabi, United Arab Emirates. The Arup Journal (2013) 2:60-73.

[31 Karanouh, A and Kerber, E. Innovations in dynamic architecture Journal of Façade Design and Engineering (2015) 3(2):185-221. DOI 10.3233/FDE-150040.

[4] Babilio, E., Miranda, R. and Fraternali, F. On the kinematics and actuation of dynamic sunscreens with tensegrity architecture. Frontiers in Materials (2019) 6:7. DOI:10.3389/ fmats.2019.00007.

[5] Balduzzi, F., Bianchini, A. and Ferrari, L. Microeolic turbines in the built environment: Influence of the installation site on the potential energy yield. Renewable Energy (2012) 5:163-174.
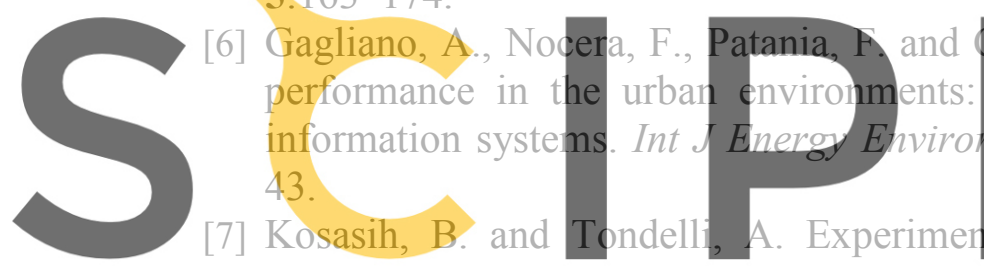
Kosasih, B. and Tondell
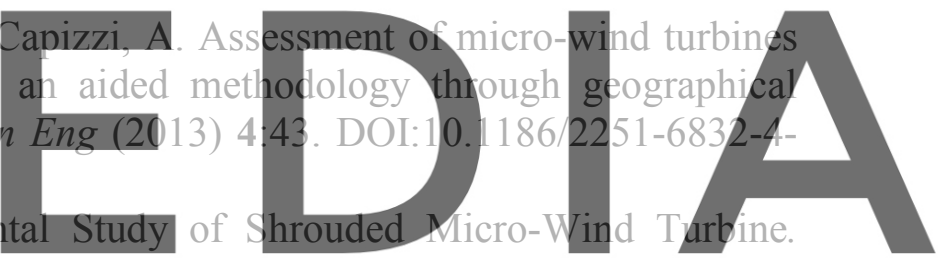
Procedia Engineering (2012) 49:92-98. DOI:10.1016/j.proeng.2012.10.116.

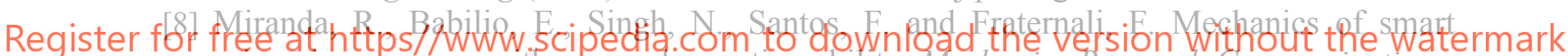
origami sunscreens with energy harvesting ability. Mechanics Research Communications, (2020) 105:103503. DOI:10.1016/j.mechrescom.2020.103503.

[9] Power Jacks, Rolaram, Electric Linear Actuators. Documentation and data available at: https://www.powerjacks.com/perch/resources/brochure/pjlab-rolaram-en-01-1b.pdf. Date accessed: 26 dec. 2018.

[10] Skelton, R. Dynamics and control of tensegrity systems. In: IUTAM Symposium on Vibration Control of Nonlinear Mechanisms and Structures, pp. 309-318. Springer Netherlands (2005).

[11] Schek, H.J. The force density method for form finding and computation of general networks. Computer Methods in Applied Mechanics and Engineering (1974) 3(1):115134. DOI 10.1016/0045-7825(74)90045-0

[12] Nagase, K. and Skelton, R.E. Network and vector forms of tensegrity system dynamics. Mechanics Research Communicastions (2014) 59:14-25.

[13] Sultan, C., Corless, M. and Skelton, R. Linear dynamics of tensegrity structures. Engineering Structures (2002) 24(6):671-685. DOI 10.1016/S0141-0296(01)00130-4.

[14] EN 1991-1-4 (2005) (English): Eurocode 1: Actions on structures, Parts 1-4: General actions. Wind actions [Authority: The European Union Per Regulation 305/2011, 
Directive 98/34/2011, Directive 98/34/EC, Directive 2004/18/EC].

[15] Santos, F., Gonçalves, P., Cismasiu, C. and Gamboa-Marrufo, M. Smart glass façade subjected to wind loadings. Structures and Buildings (2014) 167(12):743-752. DOI:10.1680/stbu.13.00011.

[16] Sanghoon, P., Yura K., Hyosub J., Jun-Young P., Naesung L. and Yongho S. Energy harvesting efficiency of piezoelectric polymer film with graphene and metal electrodes. Scientific Reports (2017) 7:17290. DOI: 10.1038/s41598-017-17791-3.

[17] Reza Moheimani, S.O. and Fleming, A.J. Piezoelectric Transducers for Vibration Control and Damping. Springer, London (2006). DOI 10.1007/1-84628-332-9

[18] Holloway F.C. (1997) Material Characterization of Poly (vinylidene Fluoride): a thin Film Piezoelectric Polymer. Montana State University- Bozeman, thesis for the degree Of Master of Science in Mechanical Engineering, $252 \mathrm{pp}$

[19] Grzybek, D. Piezoelectric generators: materials and structures. Pomiary Automatyka Robotyka (2013) 10:123-129. DOI: 10.14313/PAR 200/123.

[20] Fabbrocino, F., Farina, I. and Modano, M. Loading noise effects on the system identification of composite structures by dynamic tests with vibrodyne. Composites Part B-Engineering (2017) 115:376-383. DOI:10.1016/j.compositesb.2016.09.032
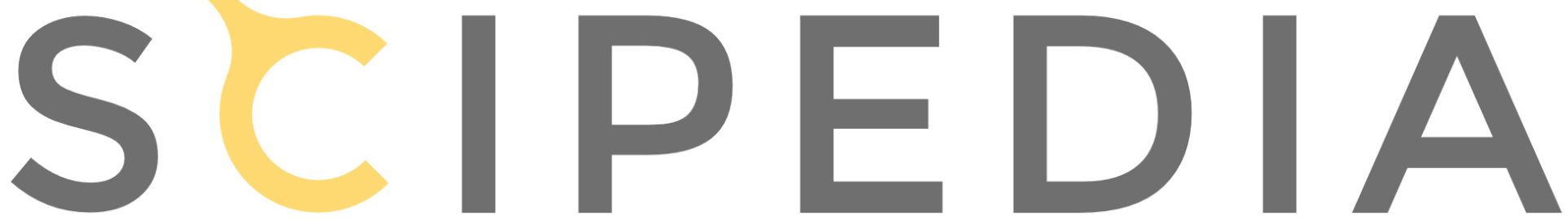

Register for free at https//www.scipedia.com to download the version without the watermark 